La Plata-Th $00 / 11$

November 2000

\title{
An alternative formulation of classical electromagnetic duality
}

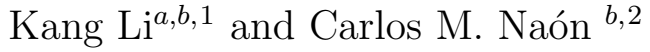 \\ ${ }^{a}$ Department of Physics, Zhejiang University, Hangzhou, 310027, P.R. China \\ ${ }^{b}$ Instituto de Física La Plata, Departamento de Física, Facultad de Ciencias Exactas, \\ Universidad Nacional de La Plata , CC 67,1900 La Plata, Argentina
}

\begin{abstract}
By introducing a doublet of electromagnetic four dimensional vector potentials, we set up a manifestly Lorentz covariant and $S O(2)$ duality invariant classical field theory of electric and magnetic charges. In our formulation one does not need to introduce the concept of Dirac string.
\end{abstract}

Keywords: electromagnetic duality, classical field theory, monopoles.

PACS: 03.50.De, 11.30.-j, 14.80.Hv

\footnotetext{
${ }^{1}$ kangli@mail.hz.zj.cn

${ }^{2}$ naon@venus.fisica.unlp.edu.ar
} 


\section{Introduction}

Recently there has been much interest in the study of electromagnetic (EM) duality, perhaps due to its role in unifying theories [1]. In particular, the construction of a fundamental "M-theory" in eleven dimensions, based on extended objects called branes, relies on the implementation of an EM duality. ¿From Maxwell's equations we know that general EM duality implies the existence of magnetic charge (monopole) and currents. However, when considering the quantum dynamics of particles carrying both electric and magnetic charges (dyons) one faces the lack of a naturally defined classical field theory despite of the fact that a consistent quantum field theory does exist [2]. This issue was analyzed in recent contributions by considering certain generalized Dirac-strings [4] (See also [5] for a formulation of dual quantum electrodynamics based on the original string-dependent action of Dirac and Schwinger). In [6] a non local Lagrangian formalism was proposed whose quantized version allowed to compute probability amplitudes for charge-monopole scattering [7]. There were also some other recent works that focused on different aspects of classical electromagnetism such as its connection to General Relativity [8], consistency conditions [9] [10], etc.

The aim of this work is to present and explore an alternative formulation of EM duality, in a classical context, which avoids the concept of Dirac-string. This classical field theory is based on the introduction of a doublet of 4-dimensional vector potentials that have no singularity around the magnetic monopoles. It posses manifest Lorentz covariance and $S O(2)$ duality symmetry. The main advantage of our formulation is that, since the gauge fields are regular, one expects that their quantization will be straightforward.

The paper is organized as follows. In the next section we give a brief review of classical EM duality and explain why one needs to introduce the Dirac monopole and the Dirac string around the monopole. In the third section we describe in some detail our formulation. In section 4 , in order to illustrate the physical content of this model and allow comparison with the Dirac-string formulation, we solve the classical equations of motion for some specific static and non-static cases. In section 5 we derive a generalized Lorentz force formula and show how to get the electric charge quantization condition. Finally, in section 6 we display the Lagrangian form of our formulation and present our main conclusions. 


\section{$2 \quad$ EM duality and the Dirac string}

Let us start by recalling the basic concepts related to EM duality and the Dirac monopole. In terms of the electric field $\mathbf{E}$, the magnetic induction $\mathbf{B}$, the charge and current densities $\rho_{e}$ and $\mathbf{j}_{\mathbf{e}}$, Maxwell's equations read

$$
\begin{aligned}
& \nabla \cdot \mathbf{E}=\rho_{e}, \quad \nabla \times \mathbf{B}=\frac{\partial \mathbf{E}}{\partial t}+\mathbf{j}_{\mathbf{e}} \\
& \nabla \cdot \mathbf{B}=0, \quad \nabla \times \mathbf{E}=-\frac{\partial \mathbf{B}}{\partial t} .
\end{aligned}
$$

where we set $c=\hbar=1$ and $\mu_{0}=\varepsilon_{0}=1$. As usual, one introduces the electric scalar potential $\phi_{e}$ and the magnetic vector potential $\mathbf{A}_{\mathbf{m}}$ such that

$$
\begin{gathered}
\mathbf{E}=-\nabla \phi_{e}-\frac{\partial \mathbf{A}_{\mathbf{m}}}{\partial t} \\
\mathbf{B}=\nabla \times \mathbf{A}_{\mathbf{m}} \\
A_{\mu}=\left(\phi_{e},-\mathbf{A}_{\mathbf{m}}\right), \quad x_{\mu}=(t,-\mathbf{x}), \quad J_{\mu}^{e}=\left(\rho_{e},-\mathbf{j}_{\mathbf{e}}\right) .
\end{gathered}
$$

Thus, in terms of the electro-magnetic field tensor

$$
F_{\mu \nu}=\partial_{\mu} A_{\nu}-\partial_{\nu} A_{\mu}
$$

Maxwell's equations (2.1) and (2.2) can be written in a manifestly Lorentz covariant form as

$$
\partial^{\mu} F_{\mu \nu}=J_{\nu}^{e}
$$

and

$$
\partial_{\sigma} F_{\mu \nu}+\partial_{\mu} F_{\nu \sigma}+\partial_{\nu} F_{\sigma \mu}=0
$$

Furthermore, if we define the dual of the electromagnetic field tensor as

$$
{ }^{*} F_{\mu \nu}=\frac{1}{2} \epsilon_{\mu \nu \rho \sigma} F^{\rho \sigma}
$$

Maxwell's equations become

$$
\partial^{\mu} F_{\mu \nu}=J_{\nu}^{e}, \quad \partial^{\mu *} F_{\mu \nu}=0 .
$$

In the source free case, i.e. $J_{\mu}^{e}=\left(\rho_{e},-\mathbf{j}_{\mathbf{e}}\right)=0$, the duality symmetry of the above equations becomes apparent. Indeed, it is easy to show that under the dual transformation 
given by $F_{\mu \nu} \rightarrow{ }^{*} F_{\mu \nu}, \quad{ }^{*} F_{\mu \nu} \rightarrow{ }^{* *} F_{\mu \nu}=-F_{\mu \nu}$, the equations (2.10) are unchanged. In fact, taking into account that

$$
F_{\mu \nu}=\left(\begin{array}{cccc}
0 & E_{x} & E_{y} & E_{z} \\
-E_{x} & 0 & B_{z} & -B_{y} \\
-E_{y} & -B_{z} & 0 & B_{x} \\
-E_{z} & B_{y} & -B_{x} & 0
\end{array}\right)
$$

and

$$
{ }^{*} F_{\mu \nu}=\left(\begin{array}{cccc}
0 & B_{x} & B_{y} & B_{z} \\
-B_{x} & 0 & -E_{z} & E_{y} \\
-B_{y} & E_{z} & 0 & -E_{x} \\
-B_{z} & -E_{y} & E_{x} & 0
\end{array}\right),
$$

one can readily convince oneself that the dual transformation is equivalent to performing the following replacement in Maxwell's equations:

$$
\mathbf{E} \rightarrow \mathbf{B}, \quad \mathbf{B} \rightarrow-\mathbf{E}
$$

The duality symmetry is immediately broken if a non-zero electric current $J_{\mu}^{e}$ enters the theory, unless a non-zero magnetic current $J_{\mu}^{m}=\left(\rho_{m},-\mathbf{j}_{\mathbf{m}}\right)$ is also introduced. When both electric and magnetic sources are included Maxwell's equations read

$$
\begin{array}{cc}
\nabla \cdot \mathbf{E}=\rho_{e}, & \nabla \times \mathbf{B}=\mathbf{j}_{\mathbf{e}}+\frac{\partial \mathbf{E}}{\partial t}, \\
\nabla \cdot \mathbf{B}=\rho_{m}, & \nabla \times \mathbf{E}=-\mathbf{j}_{\mathbf{m}}-\frac{\partial \mathbf{B}}{\partial t}
\end{array}
$$

and both electric and magnetic currents are conserved, i.e. they satisfy

$$
\begin{gathered}
\partial^{\mu} J_{\mu}^{e}=0 \\
\partial^{\mu} J_{\mu}^{m}=0 .
\end{gathered}
$$

Maxwell's equations and currents conservation conditions above are obviously invariant under the dual transformation, i.e. they are invariant under the replacement (2.13) together with

$$
\rho_{e} \rightarrow \rho_{m}, \quad \rho_{m} \rightarrow-\rho_{e}, \quad \mathbf{j}_{\mathbf{e}} \rightarrow \mathbf{j}_{\mathbf{m}}, \quad \mathbf{j}_{\mathbf{m}} \rightarrow-\mathbf{j}_{\mathbf{e}}
$$

At this point the question arises about how to formulate Maxwell's duality theory in the presence of sources, in a Lorentz covariant way. It is clear that one needs to modify the Maxwell's equation (2.10) in the form

$$
\partial^{\mu} F_{\mu \nu}=J_{\nu}^{e}, \quad \partial^{\mu *} F_{\mu \nu}=J_{\nu}^{m} .
$$


But, as we know, if the field tensor $F_{\mu \nu}$ is defined by equation (2.6) then the second equation of (2.10) (Bianchi identity) always holds unless the potential $A_{\mu}$ have a singularity somewhere. In other words, if we compute the magnetic flux through a sphere $S^{2}$ which surrounds the monopole with magnetic charge $g$, we shall always get

$$
\iint_{s^{2}} \mathbf{B} \cdot d \mathbf{S}=\iint_{s^{2}}(\nabla \times \mathbf{A}) \cdot d \mathbf{S}=0
$$

although, due to the existence of the monopole, we must have a flux equal to $g$. So, as is well-known, the way out of this problem is to allow A to have a singularity somewhere on the sphere. This argument can be used for any radius of the spheres surrounding the monopole, so by increasing it from zero to infinity we conclude that the monopole has attached a line of singularities. This line is called the Dirac string [3], and this kind of monopole is also called Dirac monopole. The Dirac string is not a physical observable and one should not be able to measure it, so the orientation of the Dirac string can be chosen arbitrarily (different orientations correspond to different gauge choices). As a by product of the Dirac string one can get the quantization condition for the electric charge. However, as will be shown in section 5, the Dirac string is not necessary to obtain that condition.

\section{$3 \quad$ EM duality without Dirac strings}

In this section we will present an alternative formulation of EM duality without the use of Dirac strings. Besides $\phi_{e}$ and $\mathbf{A}_{\mathbf{m}}$ defined in the previous section, let us now introduce also a scalar potential $\phi_{m}$ associated to the magnetic field and a vector potential $\mathbf{A}_{\mathbf{e}}$ associated to the electric field. The electric field $\mathbf{E}$ and the magnetic induction $\mathbf{B}$ are then expressed as:

$$
\begin{gathered}
\mathbf{E}=-\nabla \phi_{e}-\frac{\partial \mathbf{A}_{\mathbf{m}}}{\partial t}+\nabla \times \mathbf{A}_{\mathbf{e}} \\
\mathbf{B}=\nabla \phi_{m}+\frac{\partial \mathbf{A}_{\mathbf{e}}}{\partial t}+\nabla \times \mathbf{A}_{\mathbf{m}} .
\end{gathered}
$$

Now we substitute these definitions in Maxwell's equation (2.14) and (2.15), and use the Lorentz gauge given by:

$$
\nabla \cdot \mathbf{A}_{\mathbf{m}}+\frac{\partial \phi_{e}}{\partial t}=0, \quad \nabla \cdot \mathbf{A}_{\mathbf{e}}+\frac{\partial \phi_{m}}{\partial t}=0
$$


Thus we get

$$
\begin{aligned}
& \frac{\partial^{2}}{\partial t^{2}} \phi_{e}-\nabla^{2} \phi_{e}=\rho_{e} \\
& \frac{\partial^{2}}{\partial t^{2}} \mathbf{A}_{\mathbf{m}}-\nabla^{2} \mathbf{A}_{\mathbf{m}}=\mathbf{j}_{\mathbf{e}} \\
& \frac{\partial^{2}}{\partial t^{2}} \phi_{m}-\nabla^{2} \phi_{m}=-\rho_{m}, \\
& \frac{\partial^{2}}{\partial t^{2}} \mathbf{A}_{\mathbf{e}}-\nabla^{2} \mathbf{A}_{\mathbf{e}}=-\mathbf{j}_{\mathbf{m}}
\end{aligned}
$$

At this point we define

$$
\begin{aligned}
& A_{\mu}^{1}=\left(\phi_{e},-\mathbf{A}_{\mathbf{m}}\right), \quad A^{\mu 1}=\left(\phi_{e}, \mathbf{A}_{\mathbf{m}}\right), \\
& A_{\mu}^{2}=\left(\phi_{m},-\mathbf{A}_{\mathbf{e}}\right), \quad A^{\mu 2}=\left(\phi_{m}, \mathbf{A}_{\mathbf{e}}\right),
\end{aligned}
$$

and

$$
J_{\mu}^{1}=J_{\mu}^{e}=\left(\rho_{e},-\mathbf{j}_{\mathbf{e}}\right), \quad J_{\mu}^{2}=J_{\mu}^{m}=\left(\rho_{m},-\mathbf{j}_{\mathbf{m}}\right) .
$$

Equations in (3.4) can then be written as

$$
\begin{gathered}
\partial_{\mu} \partial^{\mu} A_{\nu}^{1}=J_{\nu}^{1} \\
\partial_{\mu} \partial^{\mu} A_{\nu}^{2}=-J_{\nu}^{2}
\end{gathered}
$$

In analogy to formula (2.6) we also write

$$
F_{\mu \nu}^{I}=\partial_{\mu} A_{\nu}^{I}-\partial_{\nu} A_{\mu}^{I}, \quad I=1,2 .
$$

Taking into account that Lorentz gauge (3.3) can now be expressed as

$$
\partial^{\mu} A_{\mu}^{I}=0
$$

we have

$$
\partial^{\mu} F_{\mu \nu}^{I}=g^{I I^{\prime}} J_{\nu}^{I^{\prime}}
$$

where

$$
g^{I I^{\prime}}=\left(\begin{array}{cc}
1 & 0 \\
0 & -1
\end{array}\right) .
$$

This is our first non trivial result. Equation (3.11) is the new version of Maxwell's equations, which have manifest Lorentz symmetry and $\mathrm{SO}(2)$ dual symmetry. The currents $J_{\mu}^{I}$ are conserved currents. In our approach this conservation reads

$$
\partial^{\nu} J_{\nu}^{I} \propto \partial^{\nu} \partial^{\mu} F_{\mu \nu}^{I}=0
$$


which are equivalent to equations (2.16) and (2.17).

We should mention that the general gauge transformation in this formalism is given by $A_{\mu}^{I} \rightarrow A_{\mu}^{I}+\partial_{\mu} \chi^{I}$. It is easy to check that the fields $\mathbf{E}, \mathbf{B}$ as well as the field tensors in (3.10) and Maxwell's equations (3.11) are all invariant under such a transformation.

Let us also stress that in the expressions above neither $F_{\mu \nu}^{1}$ nor $F_{\mu \nu}^{2}$ have the same matrix form given by (2.11) and (2.12). In order to facilitate comparison between our formalism and the one depicted in section 2 it is useful to define

$$
\begin{aligned}
& \mathcal{F}_{\mu \nu}=F_{\mu \nu}^{1}+{ }^{*} F_{\mu \nu}^{2} \\
& \widetilde{\mathcal{F}}_{\mu \nu}={ }^{*} F_{\mu \nu}^{1}-F_{\mu \nu}^{2}
\end{aligned}
$$

where $\widetilde{\mathcal{F}}_{\mu \nu}$ can be viewed as a new dual field tensor that, as we shall see, is specially adequate to express the duality symmetry in a compact fashion. The matrix form for $\mathcal{F}_{\mu \nu}$ is exactly the same as the form in (2.11). On the other hand the newly introduced $\widetilde{\mathcal{F}}_{\mu \nu}$ has a matrix form equal to the one corresponding to ${ }^{*} F_{\mu \nu}$ in the previous section (equation (2.12)). Of course the electric and magnetic fields in this new expressions are given by (3.1) and (3.2) whereas the older ones were determined by (2.3) and (2.4). Since the vector potentials in our formalism have no singularities one has $\partial^{\mu *} F_{\mu \nu}^{I}=0$, so Maxwell's equations can also be written as

$$
\begin{aligned}
& \partial^{\mu} \mathcal{F}_{\mu \nu}=\partial_{\mu} F_{\mu \nu}^{1}=J_{\nu}^{1} \\
& \partial^{\mu} \widetilde{\mathcal{F}}_{\mu \nu}=-\partial_{\mu} F_{\mu \nu}^{2}=J_{\nu}^{2}
\end{aligned}
$$

This couple of equations is obviously invariant under the dual transformation $\mathcal{F}_{\mu \nu} \rightarrow$ $\widetilde{\mathcal{F}}_{\mu \nu}, \quad \widetilde{\mathcal{F}}_{\mu \nu} \rightarrow-\mathcal{F}_{\mu \nu}, \quad J_{\mu}^{1} \rightarrow J_{\mu}^{2}, \quad J_{\mu}^{2} \rightarrow-J_{\mu}^{1}$, which are equivalent to $\mathbf{E} \rightarrow-\mathbf{B}$ and $\mathbf{B} \rightarrow \mathbf{E}$. This duality symmetry is called special duality symmetry 11]. In fact, (3.15) has a more general duality symmetry under the following transformations:

$$
\begin{aligned}
\left(\begin{array}{c}
\mathcal{F}^{\prime}{ }_{\mu \nu} \\
\widetilde{\mathcal{F}}_{\mu \nu}^{\prime}
\end{array}\right) & =\left(\begin{array}{ll}
a & c \\
b & d
\end{array}\right)\left(\begin{array}{c}
\mathcal{F}_{\mu \nu} \\
\widetilde{\mathcal{F}}_{\mu \nu}
\end{array}\right), \\
\left(\begin{array}{c}
J^{\prime 1} \\
J^{\prime 2} \\
\mu
\end{array}\right) & =\left(\begin{array}{ll}
a & c \\
b & d
\end{array}\right)\left(\begin{array}{c}
J_{\mu}^{1} \\
J_{\mu}^{2}
\end{array}\right)
\end{aligned}
$$

with $a d-b c \neq 0$. This symmetry should also hold for Maxwell's equations (2.14) and (2.15). Indeed, invariance of these equations under the change

$$
\left(\begin{array}{l}
\mathbf{E}^{\prime} \\
\mathbf{B}^{\prime}
\end{array}\right)=\left(\begin{array}{ll}
a & c \\
b & d
\end{array}\right)\left(\begin{array}{l}
\mathbf{E} \\
\mathbf{B}
\end{array}\right)
$$


yields $a=d$ and $b=-c$. Moreover, if we impose that the energy density and the Poynting vector are also invariant under this transformation we get $a^{2}+b^{2}=1$. It is then natural to introduce an angle $\alpha$ such that $a=\cos \alpha$ and $b=\sin \alpha$. Hence the general duality transformation matrix coincides with the general rotation matrix in two dimensions. Thus it becomes apparent that the general EM duality symmetry is the $\mathrm{SO}(2)$ symmetry.

Let us also mention that it would be desirable to get equation (3.15) from a variational principle, exactly as it is done in the absence of monopoles [12] (see also [13]). This issue will be considered in section 6 .

It is also important to stress that our two potentials formulation can be recast in terms of one unique vector field $\mathcal{A}_{\mu}$ defined as

$$
\mathcal{A}_{\mu}=A_{\mu}^{1}+{ }^{*} A_{\mu}^{2}
$$

where ${ }^{*} A_{\mu}^{I}$ are defined through

$$
\partial_{\mu}{ }^{*} A_{\nu}^{I}=\frac{1}{2} \epsilon_{\mu \nu}{ }^{\alpha \beta} \partial_{\alpha} A_{\beta}^{I}
$$

The field tensor $\mathcal{F}_{\mu \nu}$ can then be expressed as

$$
\mathcal{F}_{\mu \nu}=\partial_{\mu} \mathcal{A}_{\nu}-\partial_{\nu} \mathcal{A}_{\mu}
$$

This tensor has the same form as in equation (2.6), but here the dual of $\mathcal{F}_{\mu \nu}$ coincides with $\widetilde{\mathcal{F}}_{\mu \nu}$, it does not satisfy an equation of the form (2.9). As a consequence the equations in (2.10) are now replaced by equations (3.15). Let us also stress that after quantization the field $\mathcal{A}_{\mu}$ will be associated to the photon.

\section{Application to specific electromagnetic systems}

In this section we will give some explicit solutions for specific static systems in the presence of both electric and magnetic sources. At the end we will briefly comment on the formal solutions for the general non-static case.

In a static situation the Maxwell's equation (3.4) becomes

$$
\begin{aligned}
& \nabla^{2} \phi_{e}=-\rho_{e}(\mathbf{x}), \quad \nabla^{2} \phi_{m}=\rho_{m}(\mathbf{x}), \\
& \nabla^{2} \mathbf{A}_{\mathbf{m}}=-\mathbf{J}_{\mathbf{e}}(\mathbf{x}), \quad \nabla^{2} \mathbf{A}_{\mathbf{e}}=\mathbf{J}_{\mathbf{m}}(\mathbf{x})
\end{aligned}
$$

For simplicity, we shall consider a dyon with electric charge $q$ and magnetic charge $g$ placed at the origin of the coordinate system:

$$
\begin{aligned}
& \nabla^{2} \phi_{e}=-q \delta(\mathbf{x}), \quad \nabla^{2} \phi_{m}=g \delta(\mathbf{x}) \\
& \nabla^{2} \mathbf{A}_{I}=0, \quad I=e, m .
\end{aligned}
$$


The solutions in boundless space are

$$
\begin{aligned}
& \phi_{e}=\frac{1}{4 \pi} \frac{q}{r}, \quad \phi_{m}=-\frac{1}{4 \pi} \frac{g}{r}, \\
& \mathbf{A}_{I}=0, \quad I=e, m .
\end{aligned}
$$

Then from (3.1) and (3.2), the field strengths are given by

$$
\begin{aligned}
\mathbf{E} & =\frac{q \mathbf{r}}{4 \pi r^{3}} \\
\mathbf{B} & =\frac{g \mathbf{r}}{4 \pi r^{3}} .
\end{aligned}
$$

Let us emphasize that these simple solutions are obtained without using the concept of Dirac string.

As a second example we now consider a steady current of dyons in a circular loop. To be definite let us place the circle with radius $R$ in the $x y$ plane and use spherical coordinates $r, \theta$ and $\varphi$. The only non-vanishing components of the current densities correspond to the $\varphi$ direction:

$$
J_{\varphi}^{I}(r, \theta)=J^{I} \delta(\cos (\theta)) \frac{\delta(r-R)}{R}, \quad I=e, m .
$$

where $J$ is the number of dyons passing through a cross-section in the unit of time, $J^{e}=q J$ and $J^{m}=g J$. Since $J^{I}$ has only $\varphi$-components the same will happen with $\mathbf{A}^{I}$. Therefore the solutions of Maxwell's equation (4.1) for this case read

$$
A_{\varphi}^{I}(r, \theta)=\frac{J^{I} R}{\pi \sqrt{R^{2}+r^{2}+2 R r \sin \theta}}\left(\frac{\left(2-k^{2}\right) K(k)-2 E(k)}{k^{2}}\right)
$$

where

$$
k^{2}=\frac{4 R r \sin \theta}{R^{2}+r^{2}+2 R r \sin \theta}
$$

and $E(k)$ and $K(k)$ are elliptic integrals.

For small $k$, one gets

$$
A_{\varphi}^{I}=\frac{J^{I} R^{2}}{4} \frac{r \sin \theta}{\left(R^{2}+r^{2}+2 R r \sin \theta\right)^{3 / 2}}
$$

Noticing that in this example one has $\phi_{e}=\phi_{m}=0$, we obtain $\mathbf{E}=\nabla \times \mathbf{A}_{\mathbf{e}}, \mathbf{B}=\nabla \times \mathbf{A}_{\mathbf{m}}$. This means that for $k$ small (which corresponds to $R>>$, or $r>>R$, or $\theta<<1$ ), we 
find

$$
\begin{aligned}
& B_{r}=\frac{q J \cos \theta}{4} \frac{2 R^{2}+2 r^{2}+R r \sin \theta}{\left(R^{2}+r^{2}+2 R r \sin \theta\right)^{5 / 2}} \\
& B_{\theta}=-\frac{q J \sin \theta}{4} \frac{2 R^{2}+2 r^{2}+R r \sin \theta}{\left(R^{2}+r^{2}+2 R r \sin \theta\right)^{5 / 2}} \\
& B_{\varphi}=0
\end{aligned}
$$

and

$$
\begin{aligned}
& E_{r}=\frac{g J \cos \theta}{4} \frac{2 R^{2}+2 r^{2}+R r \sin \theta}{\left(R^{2}+r^{2}+2 R r \sin \theta\right)^{5 / 2}} \\
& E_{\theta}=-\frac{g J \sin \theta}{4} \frac{2 R^{2}+2 r^{2}+R r \sin \theta}{\left(R^{2}+r^{2}+2 R r \sin \theta\right)^{5 / 2}} \\
& E_{\varphi}=0 .
\end{aligned}
$$

When the magnetic charge vanishes $(g=0)$, the above results coincide with the wellknown expressions obtained for an electric charge moving steadily in a circular loop. On the other hand, for $g \neq 0$, it is not simple to evaluate the fields using the concept of Dirac strings.

For completeness let us now display the formal solutions for general non-static distributions:

$$
\rho_{I}=\rho_{I}(\mathbf{x}, \mathbf{t}), \quad \mathbf{J}_{I}=\mathbf{J}_{I}(\mathbf{x}, \mathbf{t}), \quad I=1,2 \text {. represent } I=e, m .
$$

In principle this problem can be analyzed by means of the retarded potential method, exactly as it is done in $g=0$ classical electrodynamics [14]. The solution of equations (3.4) is then given by

$$
\begin{aligned}
& \phi_{I}(\mathbf{x}, t)=\frac{1}{4 \pi} \int \frac{g^{I I^{\prime}} \rho_{I^{\prime}}\left(\mathbf{x}^{\prime}, t-r\right)}{r} d^{3} x^{\prime} \\
& \mathbf{A}_{I}(\mathbf{x}, t)=\frac{1}{4 \pi} \int \frac{g^{I I^{\prime}} \mathbf{J}_{I^{\prime}}\left(\mathbf{x}^{\prime}, t-r\right)}{r} d^{3} x^{\prime},
\end{aligned}
$$

where $r=\left|\mathbf{x}-\mathbf{x}^{\prime}\right|$ and $g^{I I^{\prime}}$ is given by the definition below the equation (3.11), then the field strengths are

$$
\begin{aligned}
& \mathbf{E}(\mathbf{x}, t)=\frac{1}{4 \pi} \int \rho_{e}\left(\mathbf{x}^{\prime}, t-r\right) \frac{\mathbf{r}}{r^{3}} d^{3} x^{\prime} \\
& +\frac{1}{4 \pi} \int \mathbf{J}_{m}\left(\mathbf{x}^{\prime}, t-r\right) \times \frac{\mathbf{r}}{r^{3}} d^{3} x^{\prime}-\frac{1}{4 \pi} \int \frac{1}{r} \frac{\partial \mathbf{J}_{m}\left(\mathbf{x}^{\prime}, t-r\right)}{\partial t} d^{3} x^{\prime},
\end{aligned}
$$


and

$$
\begin{aligned}
& \mathbf{B}(\mathbf{x}, t)=\frac{1}{4 \pi} \int \rho_{m}\left(\mathbf{x}^{\prime}, t-r\right) \frac{\mathbf{r}}{r^{3}} d^{3} x^{\prime} \\
& -\frac{1}{4 \pi} \int \mathbf{J}_{e}\left(\mathbf{x}^{\prime}, t-r\right) \times \frac{\mathbf{r}}{r^{3}} d^{3} x^{\prime}+\frac{1}{4 \pi} \int \frac{1}{r} \frac{\partial \mathbf{J}_{e}\left(\mathbf{x}^{\prime}, t-r\right)}{\partial t} d^{3} x^{\prime} .
\end{aligned}
$$

The solutions for the general static case is obtained from the expressions above by omitting the time derivative terms and of course one shoud also omit $t$ and $t-r$ in the corresponding arguments.

\section{Generalized Lorentz force and the electric charge quantization condition}

As it is well-known a particle with electric charge $q$ moving in the electromagnetic field will be subjected to a Lorentz force:

$$
\mathbf{F}=q \mathbf{E}+q \mathbf{v} \times \mathbf{B}
$$

where $\mathbf{v}$ is the velocity of the particle. From the dual symmetry, we know that if a particle with magnetic charge $g$ moves in an electromagnetic field, it will also feel a force given by

$$
\mathbf{F}=g \mathbf{B}-g \mathbf{v} \times \mathbf{E}
$$

Now, if we consider a dyon moving in the electromagnetic field, it will gain a generalized Lorentz force:

$$
\mathbf{F}=q(\mathbf{E}+\mathbf{v} \times \mathbf{B})+g(\mathbf{B}-\mathbf{v} \times \mathbf{E})
$$

and the equation of motion in a covariant form reads

$$
m \ddot{x^{\mu}}=\left(q \mathcal{F}^{\mu \nu}+g \widetilde{\mathcal{F}}^{\mu \nu}\right) \dot{x_{\nu}}
$$

When $g=0$, we have $\mathcal{F}_{\mu \nu} \rightarrow F_{\mu \nu}$, equation (5.4) returns to the well know result in electrodynamics.

Now let us consider a particle with electric charge $q$ moving in the field produced by a monopole with magnetic charge $g$. We place a very heavy monopole at the origin of the coordinate system and use for $\mathbf{B}$ the solution obtained in the previous section (for the static case), such that the equation of motion of the electric charge is

$$
m \frac{d^{2} \mathbf{x}}{d t^{2}}=\frac{q g}{4 \pi} \mathbf{v} \times \frac{\mathbf{r}}{r^{3}}
$$


It is easily shown that the kinetic energy and the total angular momentum are constants of motion of this system. Indeed, through elementary manipulations one can show that

$$
\frac{d}{d t}(\mathbf{L}-\kappa \hat{\mathbf{r}})=0
$$

where $\kappa=\frac{q g}{4 \pi}$ and $\mathbf{L}=\mathbf{r} \times m \mathbf{v}$ is the orbital angular momentum. Thus we find the following constant of motion,

$$
\mathbf{J}=\mathbf{L}-\kappa \hat{\mathbf{r}}
$$

This means that the total angular momentum of the system must be identified with $\mathbf{J}$ whereas the second term $(-\kappa \hat{\mathbf{r}})$ is nothing but the angular momentum of the electromagnetic field. Since we have decomposed $\mathbf{J}$ into two orthogonal parts one immediately obtains

$$
\mathbf{J}^{2}=\mathbf{L}^{2}+\kappa^{2}
$$

and now it is obvious that quantization of the total and orbital angular momentum (via equation (5.6)) translates into the Dirac quantization condition:

$$
\kappa=\frac{1}{2} \hbar \Rightarrow q g=2 \pi n, \quad n=1,2, \cdots \quad(\hbar=1) .
$$

An immediate corollary is that if a single monopole with magnetic charge $g$ exists, then the electric charge is quantized in units of $2 \pi / g$. If we consider a dyon with electric and magnetic charge $\left(q_{1}, g_{1}\right)$ moving in the field of another dyon with charges $\left(q_{2}, g_{2}\right)$, we can derive the new electromagnetic angular momentum which reads

$$
\mathbf{L}_{\mathbf{e m}}=\frac{q_{2} g_{1}-q_{1} g_{2}}{4 \pi} \frac{\mathbf{r}}{r}
$$

and the corresponding quantization condition is

$$
q_{1} g_{2}-q_{2} g_{1}=2 \pi n, \quad n=1,2, \cdots
$$

Therefore we were able to obtain the electric charge quantization condition without using the concept of Dirac string.

\section{Final remarks}

We end this work by making some remarks concerning the Lagrangian formulation. According to the description of EM duality presented in this paper, the field equations are 
given by equation (3.11). These equations can be derived from the following Lagrangian density

$$
\mathcal{L}=\frac{1}{4}\left(\widetilde{\mathcal{F}}_{\mu \nu}\right)^{2}-\mathcal{A}_{\mu} J^{\mu 1}-\widetilde{\mathcal{A}}_{\mu} J^{\mu 2},
$$

where $\mathcal{A}_{\mu}$ is given by $(3.19), \widetilde{\mathcal{A}}_{\mu}=A_{\mu}^{2}-{ }^{*} A_{\mu}^{1}, \widetilde{\mathcal{F}}_{\mu \nu}=-\left(\partial_{\mu} \widetilde{\mathcal{A}}_{\nu}-\partial_{\nu} \widetilde{\mathcal{A}}_{\mu}\right)$ and we have used $\left(\mathcal{F}_{\mu \nu}\right)^{2}=-\left({ }^{*} \mathcal{F}_{\mu \nu}\right)^{2}=-\left(\widetilde{\mathcal{F}}_{\mu \nu}\right)^{2}$. Employing the identities

$$
\begin{gathered}
\frac{\partial \mathcal{F}^{2}}{\partial\left(\partial_{\mu} \mathcal{A}_{\nu}\right)}=4 \mathcal{F}^{\mu \nu} \\
\frac{\partial \widetilde{\mathcal{F}}^{2}}{\partial\left(\partial_{\mu} \widetilde{\mathcal{A}}_{\nu}\right)}=-4 \widetilde{\mathcal{F}}^{\mu \nu},
\end{gathered}
$$

it is easy to show that the corresponding Euler-Lagrange equations

$$
\begin{aligned}
& \partial_{\mu}\left(\frac{\partial \mathcal{L}}{\partial\left(\partial_{\mu} \mathcal{A}_{\nu}\right)}\right)=\frac{\partial \mathcal{L}}{\partial \mathcal{A}_{\nu}} \\
& \partial_{\mu}\left(\frac{\partial \mathcal{L}}{\partial\left(\partial_{\mu} \widetilde{\mathcal{A}}_{\nu}\right)}\right)=\frac{\partial \mathcal{L}}{\partial \widetilde{\mathcal{A}}_{\nu}}
\end{aligned}
$$

give the right Maxwell's equation (3.15). Following the above depicted calculation one can see that the two-potential formalism proposed in this work is equivalent to the usual one potential one, which is consistent with the existence of only one kind of photon in the real world.

The first term of equation (6.1) is associated to the free electromagnetic field, whereas the second and third terms correspond to the interaction between field and source (currents) and the interaction between electric source and magnetic source. More explicitly, the interaction between electric and the magnetic sources can be expressed as

$$
-{ }^{*} A_{\mu}^{2} J^{\mu 1}+{ }^{*} A_{\mu}^{1} J^{\mu 2}
$$

Taking into account equation (3.20), we can formally write

$$
{ }^{*} A_{\mu}^{I}=-\frac{1}{2} \epsilon_{\mu \nu \alpha \beta} \int_{P}^{x} \partial^{\alpha} A^{\beta I} d x^{\nu}
$$

and using (4.11) (for the static case) we have

$$
A_{\mu}^{I}(x)=\frac{1}{4 \pi} \int \frac{g^{I I^{\prime}} J_{\mu}^{I^{\prime}}\left(\mathbf{x}^{\prime}\right)}{r} d^{3} x^{\prime} .
$$


Therefore we have,

$$
\begin{array}{r}
{ }^{*} A_{\mu}^{1} J^{\mu 2}=-\frac{1}{2} \epsilon_{\mu \nu \alpha \beta} \int_{P}^{x} \partial^{\alpha} A^{\beta 1}(y) d y^{\nu} \cdot J^{\mu 2}(x) \\
=-\frac{1}{8 \pi} \epsilon_{\mu \nu \alpha \beta} \int_{\Omega} J^{\beta 1}\left(\mathbf{x}^{\prime}\right)\left[\int_{P}^{x} \frac{\partial}{\partial y_{\alpha}} \frac{1}{\left|\mathbf{y}-\mathbf{x}^{\prime}\right|} d y^{\nu}\right] J^{\mu 2}(\mathbf{x}) d^{3} x^{\prime}
\end{array}
$$

which means that ${ }^{*} A_{\mu}^{1} J^{\mu 2}$ is just the non local interaction between the electric current $J^{\beta 1}$ and the magnetic current $J^{\mu 2}$. One has a similar result for the term ${ }^{*} A_{\mu}^{2} J^{\mu 1}$ which is obtained by interchanging $J^{\mu 1}$ and $J^{\mu 2}$ in the above equation.

In summary we have presented an alternative description of classical electromagnetism. Relevant features of this formulation are its manifest Lorentz covariance and its simple realization of duality symmetry. However, the main advantage is the fact that the fourvector potentials introduced in our formalism have no singularities, thus allowing a description of dyonic dynamics without Dirac strings. We think that these properties will be helpful when carrying over the quantization of the theory. We hope to report on this issue in a forthcoming article [16].

Acknowledgements We thank S. Antoci for sending us a copy of Ref. 13. We are also grateful to S. Carneiro for useful criticisms. This work was supported by the Consejo Nacional de Investigaciones Científicas y Técnicas (CONICET), Argentina. The authors also recognize the support of the Third World Academy of Sciences (TWAS).

\section{References}

[1] J. H. Schwarz and N. Seiberg, Rev. Mod. Phys.71 (1999) S112.

[2] R.A.Brandt, F.Neri and D.Zwanziger, Phys. Rev. D 19 (4) (1979) 1153.

[3] P. A. M. Dirac, Proc. R. Soc. London A133 (1931) 60.

[4] K. Lechner and P.A. Marchetti, hep-th/9906079.

K. Lechner, "A quantum field theory of dyons", hep-th/0003003.

[5] L. Gamberg and K. A. Milton, Phys. Rev. D 61 (2000) 75013. 
[6] P. C. R. Cardoso de Mello, S. Carneiro and M. C. Nemes, Phys. Lett. B 384 (1996) 197.

[7] S. Carneiro, JHEP 07 (1998) 022.

[8] J. Méndez, "Electromagnetic Duality based on Axiomatic Maxwell Equations", hepth/9911188.

[9] P. Singh and N. Dadhich, "The field equation from Newton's law of motion and absence of magnetic monopole", gr-qc/9912028.

[10] C. A. P. Galvao and J. A. Mignaco, "A consistent electromagnetic duality", hepth/0002182.

[11] S. Donev, "A new look on EM Duality. Suggestions and developments", hepth/0006208.

[12] H. Weyl, Ann. der Physik, 54, (1917) 118, (in german).

[13] B. Finzi, Rendiconti dell'Accademia Nazionale dei Lincei,12, (1952) 378, (in italian).

[14] J. D. Jackson, "Classical Electrodynamics", Ed. John Wiley, Singapore, 1999.

[15] B. Felsager, "Geometry, Particles and Fields", Springer-Verlag, New York, 1998. Graw-Hill Inc., 1980.

[16] Kang Li and Carlos M. Naón, "An alternative quantum field theory of dyons", ( in preparation ). 